Ann. Biol. anim. Bioch. Biophys., I97I, 11 (I), 4I-54.

\title{
DÉTERMINATION D'UN SYSTEME D'HISTOCOMPATIBILITÉ CHEZ LE PORC (SUS SCROFA) LE SYSTEME SL-A
}

M. VAIMAN, Christine RENARD, Ph. LAFAGE, J. AMETEAU et P. NIZZA

avec la collaboration technique de J. Lecointre

Laboratoire-Étable de Radiobiologie appliquée, (D. P. S. Commissariat à l'Énergie atomique)

78 - Jouy-en-Josas

\section{RÉSUMÉ}

A l'aide de la technique sérologique de lymphocytotoxicité, il a été mis en évidence un système d'histocompatibilité chez le Porc.

L'étude génétique dans cinq familles a montré que le système est gouverné par un seul locus complexe ou plusieurs loci très liés. Le ou les caractères présents sur les autosomes parentaux sont transmis en bloc à la descendance. On observe 4 classes de porcelets correspondant aux 4 combinaisons génétiques possibles. L'influence de ce système sur la durée de vie des allogreffes de peau entre frères est statistiquement significative, et tant par sa complexité que par son immunogénicité, il semble être l'homologue du système HL-A de l'homme ou H-2 de la souris.

\section{INTRODUCTION}

Le rejet d'une allogreffe, c'est-à-dire, d'une greffe transférée d'un individu à un autre individu de la même espèce est le résultat de l'incompatibilité tissulaire entre le donneur et le receveur. On limite les réactions de rejet par l'immunosuppression et par la recherche de la meilleure compatibilité possible entre le greffon et l'hôte. Nos essais d'allogreffes de moelle osseuse chez les porcs irradiés à doses létales nous ont conduit à entreprendre l'étude des antigènes d'histocompatibilité dans cette espèce. Dans une première étape, nous avons mis en évidence un système lymphocytaire à l'aide des techniques sérologiques qui sont les techniques de base pour l'étude du système d'histocompatibilité HL-A de l'Homme, considéré comme le système d'histocompatibilité majeur avec le système érythrocytaire ABO (DAUSSET et RAPAPORT, I968, a). Une fois individualisé, il a été possible d'étudier la transmission héréditaire de ce système à l'intérieur de fratries et de tester son rôle possible comme système d'histocompatibilité au moyen d'allogreffes cutanées. 


\section{I. - MATÉRIEL ET MÉTHODES}

\section{Animaux utilisés}

Soixante-dix porcs de race Large White, âgés de 3 à 5 mois, et 4 porcs de race Pitman Moore jeunes adultes, ont été utilisés tant pour la préparation des allosérums que pour le typage des fratries. Les porcs de race Large White représentent 5 familles achetées dans différents élevages, alors que les porcs Pitman Moove ont été élevés à Jouy-en-Josas.

\section{Méthode d'immunisation en vue de la préparation des allosérums}

La greffe de peau s'est révélée être le mode d'immunisation le plus efficace. La combinaison des greffes de peau utilisées en alternance avec des injections intradermiques de leucocytes donne également de bons résultats, par contre, les injections seules n'ont pas permis l'induction d'anticorps sériques à un titre utilisable. La greffe de peau se fait sous anesthésie générale par le fluothane, au masque. La peau est rasée, soigneusement lavée et désinfectée. Après divers essais, nous avons retenu comme points d'élection, les faces latérales du cou et du thorax. La greffe est totale, dermo-épidermique, rectangulaire et mesure de $I_{5}$ à $20 \mathrm{~cm}^{2}$. La face interne du greffon est lavée au sérum physiologique pour éliminer toute trace de sang et on réalise une hémostase aussi parfaite que possible du lit du greffon. La greffe est maintenue par des points séparés avec la soie 000 et on protège la plaie par un pansement adhésif. Aucune antibiothérapie n'est nécessaire.

\section{Choix des animaux pour l'immunisation}

Nous avons effectué des immunisations réciproques entre des couples de porcs. Pour les porcs de race Large White, dans $\mathrm{I} 7$ cas, les porcs de la paire sont frères et dans 6 cas, ils appartiennent à des fratries différentes. Les deux paires de Pitman Moore étaient des demi-frères.

Dans toutes les paires, l'identité pour le système érythrocytaire A-O a été respectée et lorsque cela a été possible pour un certain nombre d'autres facteurs appartenant à plusieurs systèmes de groupes sanguins indépendants : B, E, F, G, J, K.

Après la première greffe, le sérum de chaque porc a été testé contre les lymphocytes du donneur au $7^{\mathbf{e}}$ jour, puis une fois par semaine. Lorsque le titre est suffisant, on prélève 500 à $600 \mathrm{ml}$ de sang et le sérum est conservé congelé.

\section{Préparation des suspensions pures de lymphocytes}

La technique de préparation des lymphocytes à partir du sang périphérique a été décrite en détail (VAIMAN, RENARD et Nizza, I970). Elle permet d'obtenir une suspension lymphocytaire de 97 à 99 p. Ioo de pureté avec une viabilité de 95 à 99 p. Ioo.

Les lymphocytes sont utilisés soit frais, dans la journée, soit après avoir été conservés congelés à l'azote liquide. La congélation est réalisée selon la technique de refroidissement contrôlé (LoveLOCK et BisHOP, r959), en présence de diméthylsulfoxide. Les cellules sont conditionnées en paillettes de $0,5 \mathrm{ml}$ à la concentration de 5 à 7 ooo cellules par $\mathrm{mm}^{3}$. Après décongélation, le pourcentage de viabilité est de 80 à 90 p. I00 suivant les lots.

\section{Technique de lymphocytotoxicité}

\section{a) Importance du complément.}

La réaction consiste à mettre en présence les anticorps antilymphocytaires et les lymphocytes portant les antigènes correspondants. Les anticorps se combinent avec les antigènes de la membrane et en présence du complément, le complexe altère la membrane qui devient perméable aux colorants, comme le bleu trypan ou l'éosine. La nature du complément est très importante et comme pour l'Homme (Walford, Gallagher et SjaARDa, I964), le complément de lapin donne les meilleurs résultats tant pour la reproductibilité des tests que pour la sensibilité de la réaction. Il est nécessaire d'absorber le sérum de lapin car il présente une cytotoxicité naturelle pour les 
lymphocytes de porc. Cette cytotoxicité est attribuée à des anticorps hétérospécifiques (HERBERMAN, I969) et existe aussi pour les lymphocytes humains. On élimine ces anticorps par deux absorptions successives de 2 heures à $4^{\circ} \mathrm{C}$ à raison d'un volume de sérum frais de lapin pour un volume de globules rouges $\mathrm{A}$ et $\mathrm{O}$ mélangés de porc. Le complément ainsi absorbé est titré et conservé en paillettes de $0,5 \mathrm{ml}$ à - I $16^{\circ} \mathrm{C}$. La figure I montre les résultats du titrage d'un même lot de complément absorbé, brut, et par comparaison, les résultats obtenus pour un même système anticorps-cellules avec le complément de cobaye et du sérum de porc. Dans cet exemple, le sérum de cobaye est utilisable mais il donne souvent des résultats très variables. Quant au sérum de porc, il a toujours une activité complémentaire très faible. Le sérum de lapin donne régulièrement une lyse maximale pour une dilution au demi dans une solution de Hanks et un volume à cette dilution représente une unité $C^{\prime}$ roo par convention. Tous les tests sont faits avec 2 unités $C^{\prime}$ Ioo, soit un volume de complément pur pour un volume de sérum.

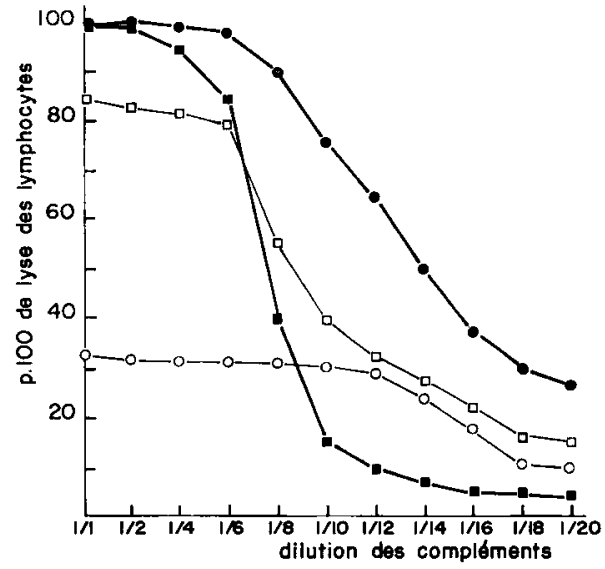

FIG. I. - Titrage des différents Compléments dans un même système Cellules-Anticorps
- complément de Lapin, absorbé
- complément de Lapin, non absorbé
complément de Cobaye
o complément de Porc

\section{b) Technique sérologique.}

La technique de lymphocytotoxicité est faite en tube pour déceler l'apparition des anticorps et leur titrage. Dans un tube à hémolyse, on mélange $0,05 \mathrm{ml}$ de sérum à la dilution convenable (après une décomplémentation de $3^{\circ}$ minutes à $5^{\circ} \mathrm{C}$ ) et $0,025 \mathrm{ml}$ de la suspension lymp hocytaire à 6 ooo par $\mathrm{mm}^{3}$. Les tubes sont mis à incuber I heure à $37^{\circ} \mathrm{C}$, au bain-marie, avec agitation tou tes les 20 minutes. On ajoute ensuite $0,05 \mathrm{ml}$ de complément et l'incubation est poursuivie pendant une heure. La réaction de lyse est révélée par addition de $0,025 \mathrm{ml}$ d'éosine (préparée extemporanément par le mélange de 3 volumes d'une solution à 5 p. Ioo d'éosine dans l'eau distillée et un volume d'une solution de Hanks rendue hypertonique par addition de $2,55 \mathrm{~g} \mathrm{de} \mathrm{NaCl}$ pour $\mathrm{roo} \mathrm{ml}$ ). On laisse agir le colorant de 3 à 5 minutes à $37^{\circ} \mathrm{C}$ et la numération des cellules lysées se fait sur une lame de Malassez, au microscope à contraste de phase.

La réaction est considérée comme négative si la lyse est inférieure à $20 \mathrm{p}$. Ioo. On note entre 20 et 40 p. $100:+$, entre 40 et 60 p. I $00:++$, entre 60 et 80 p. Ioo $:+++$ et au-dela $:++++$.

Pour le typage des porcs qui nécessite un nombre élevé de réactions, nous utilisons la technique de Terasaki et al., 1968, pratiquement sans modification. Elle donne de bons résultats pour le porc et permet d'obtenir en moyenne $95 \mathrm{p}$. Ioo de reproductibilité des tests.

\section{II. - RÉSULTATS}

\section{Apparition et évolution des anticorps}

Aucun porc ne présentait d'anticorps cytotoxiques naturels. En trois occasions, nous avons pu tester le sérum de truies multipares contre les lymphocytes de leurs 
produits et deux fois contre les cellules du verrat. Les résultats ont toujours été négatifs.

La date d'apparition des anticorps lymphocytotoxiques à un titre décelable est variable. Dans certains cas les anticorps sont présents dès le II $\mathbf{I}_{-} \mathbf{I}^{\mathbf{3}^{\mathbf{e}}}$ jour, c'està-dire, dès le rejet de la greffe primaire. Le plus souvent, il faut attendre entre le $25^{\mathrm{e}}$ et le $30^{\mathrm{e}}$ jour, soit une semaine après la greffe de rappel. Plusieurs animaux n'ont produit des anticorps qu'à la suite d'un second et même d'un troisième rappel. Deux fois, un seul des animaux de la paire a développé des anticorps à un titre utilisable et une fois aucun des deux partenaires d'une paire n'est devenu actif. Il est indiqué de prélever précocement le sérum pour éviter une multiplicité des spécificités mais il est toutefois nécessaire d'attendre que le titre soit suffisamment élevé.

En règle générale, le sérum est considéré comme utilisable s'il lyse au moins 60 à $70 \mathrm{p}$. Ioo des lymphocytes du partenaire, au pic. Les titres, définis comme la dernière dilution encore capable de lyser 30 à $40 \mathrm{p}$. Ioo des cellules varient selon les allosérums entre $\mathrm{I} / 4$ et I/I ooo. Le degré de pureté de certains d'entre eux est actuellement à l'étude, mais il semble que la plupart soient polyspécifiques.

Les figures 2 et 3 représentent respectivement des exemples de courbes d'évolution et de titration de différents sérums.

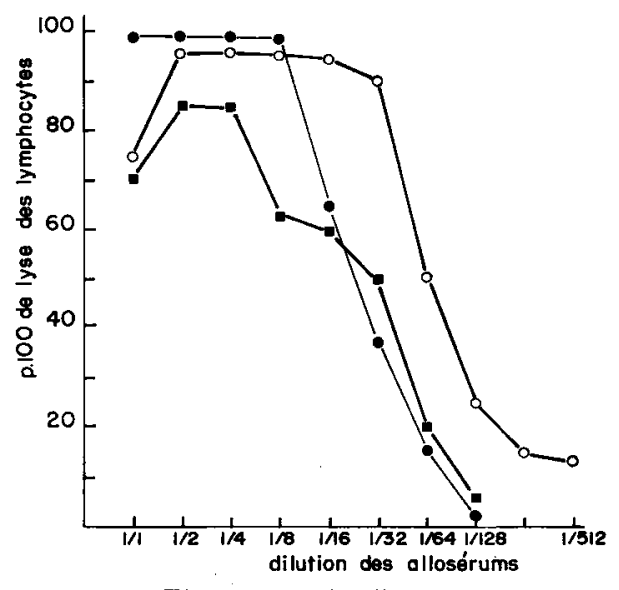

$\mathrm{F}_{\mathrm{IG}}$, 2. - Titrage de trois alloserums différents (en tubes avec 2 unités C'roo de Complément)

On remarque sur ces figures que le pic cytotoxique maximum pour un sérum donné n'atteint pas toujours Ioo p. Ioo même lorsqu'il s'agit des cellules de l'immuniseur. Le maximum de lyse ne présente pas une relation simple avec le titre, et un même sérum n'a pas le même titre ni la même activité maximale pour les cellules de différents animaux possédant la ou les spécificités correspondantes. Ceci signifie certainement que la plupart des allosérums sont polyspécifiques et on peut supposer que les épaulements ou même un second pic de certaines courbes de titration ont la même signification. Le pic d'activité n'est pas toujours retrouvé pour les concentrations sériques les plus fortes. Souvent il se situe à la dilution un demi ou même ،un quart. Ce phénomène a été souvent décrit chez 1'Homme (FERRONE, TosI, CENTIS, I967). Il peut s'agir d'un effet anticomplémentaire, mais on ne peut pas exclure la 
possibilité d'un effet protecteur pour les cellules de certaines protéines sériques ou encore des modifications du $\mathrm{pH}$ ou de concentrations salines (KoURILSKY, I966).

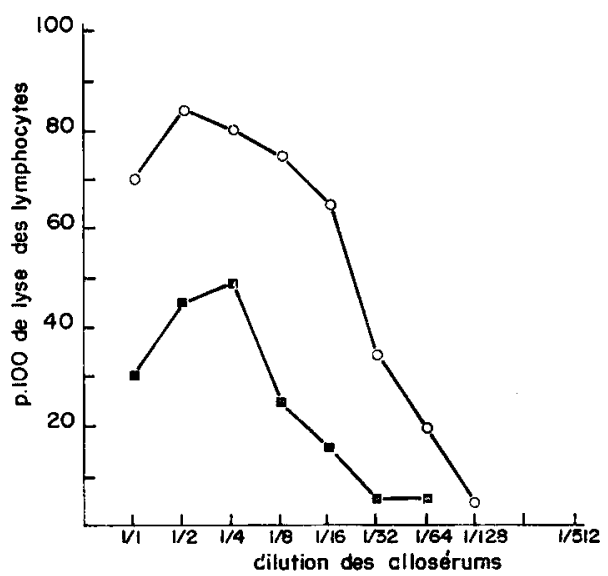

FIG. 3. - Evolution dans le temps du titre des anticorps lymphocytotoxiques chez un Porc

- sérum prélevé 30 jours après une greffe de rappel

- sérum prélevé 60 jours après un second rappel

\section{Persistance des anticorps}

Après un repos de 2 mois, le tiers des animaux n'avaient plus d'anticorps circulants à un titre décelable. Les autres porcs avaient des anticorps à un titre faible.

\section{Durée de vie des greffes de peau}

La moyenne de survies des 46 allogreffes de peau primaires chez les Large White a été de 8,5 jours avec des extrêmes de 7 à 12 jours. Bien que les greffes intra-fratries aient survécu légèrement plus longtemps que des greffes inter-fratries, la différence n'est pas significative. En fait, nous avons constaté que les critères de rejet retenus initialement, en particulier, le stade de demi-nécrose, ne permet pas de déterminer avec précision, la date réelle du rejet. Le processus de nécrose est plus ou moins rapide selon les individus et son évolution n'est pas en relation directe avec le moment où il a débuté. Pour la plupart des greffes, plusieurs stades évolutifs coexistent, ce qui rend la décision assez arbitraire. Il y a lieu de considérer les valeurs ci-dessus comme des ordres de grandeur plutôt que des résultats absolus.

Les greffes secondes sont régulièrement rejetées de manière accélérée en 4 à 6 jours et on a noté environ $30 \mathrm{p}$. Ioo de greffes "blanches". Ces dernières se caractérisent par un rejet violent sans revascularisation (STETSON et DEMOpoulos, I958). Des biopsies ont été faites sur des greffes primaires et secondaires à des temps variables et ont fait l'objet d'une étude histologique (AMETEAU, I970). Cette étude a permis de retrouver l'évolution caractéristique des allogreffes cutanées bien précisées pour toutes les espèces étudiées (MEDAwAR, 1958). 


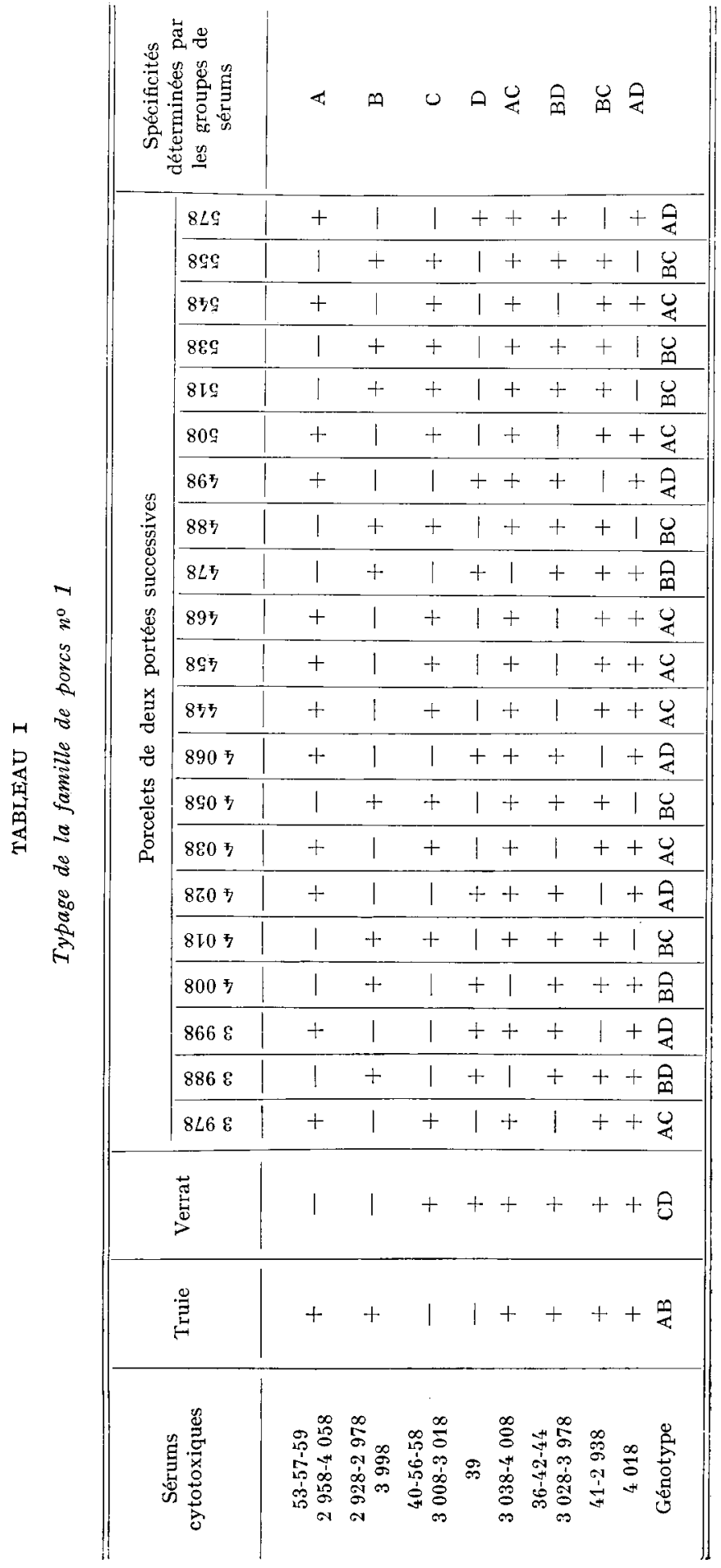




\section{Étude génétique du système lymphocytaire}

Le typage lymphocytaire a été fait sur les 70 porcs Large White, à l'aide des 46 allosérums en notre possession. Chaque animal a été testé de 4 à 8 fois, en microtechnique avec des cellules fraîches ou congelées. Les tableaux I et 2 montrent des exemples de ces typages.

La famille I (tabl. I) comprend une truie, un verrat et $2 \mathrm{I}$ porcelets issus de deux portées successives. Tous les animaux ont pu être testés. Seuls, les allosérums informatifs sont indiqués et sont groupés selon leur mode de réponse. Les deux premiers groupes ne reconnaissant que les antigènes maternels sont mutuellement exclusifs l'un de l'autre chez les porcelets et réalisent ainsi un test d'allélisme.

Il en est de même pour les deux groupes de sérums qui réagissent exclusivement avec les lymphocytes du verrat. Tous les porcelets de la famille se répartissent en quatre classes, correspondant aux quatre combinaisons théoriques possibles dans l'hypothèse d'un système antigénique gouverné par un seul locus, porté par un autosome, les caractères n'étant pas liés au sexe. Les caractères sont codominants, aucun facteur présent chez les poṛcelets n'étant absent chez les parents. Les autres groupes d'allosérums informatifs bien que moins spécifiques dans cette famille confirment entièrement la répartition antigénique.

Les résultats du typage de la famille 2 (tabl. 2) corroborent les données précé-

TABLEAU 2

Typage de la famille de porcs $n^{\circ} 2$

\begin{tabular}{|c|c|c|c|c|c|c|c|c|c|c|c|c|c|}
\hline \multirow{2}{*}{$\begin{array}{l}\text { Sérums } \\
\text { cytotoxiques }\end{array}$} & \multirow{2}{*}{$\begin{array}{l}\text { Verrat } \\
440 \mathrm{PJ}\end{array}$} & \multirow{2}{*}{$\begin{array}{l}\text { Truie } \\
418-5\end{array}$} & \multicolumn{10}{|c|}{ Porcelets } & \multirow{2}{*}{$\begin{array}{l}\text { Spécificités déter- } \\
\text { minées par les } \\
\text { groupes de sérums }\end{array}$} \\
\hline & & & 72 & $\mid 73$ & 74 & 75 & 76 & 77 & 78 & 80 & 81 & 82 & \\
\hline 3018 & + & Non typée & + & - & + & - & + & + & + & - & - & - & $\mathrm{J}$ \\
\hline $38-39-40$ & & & & & & & & & & & & & \\
\hline $\begin{array}{c}44-45-478 \\
2978-3978\end{array}$ & + & & - & + & - & + & - & - & - & + & + & + & $\mathrm{K}$ \\
\hline $3998-4028$ & & & & & & & & & & & & & \\
\hline $50-2948$ & & & & & & & & & & & & & \\
\hline 4058 & - & & - & + & + & 一 & + & 一 & + & + & + & - & L \\
\hline $69^{a}$ & - & & + & - & - & + & - & + & - & - & - & + & M \\
\hline $53-57$ & & & & & & & & & & & & & \\
\hline $2958-9398$ & + & & + & + & + & - & + & + & + & + & + & - & JL \\
\hline $42-64-3038$ & + & & + & +1 & - & + & - & $t$ & - & + & + & + & $\mathrm{KM}$ \\
\hline Génotypes & JK & $\mathrm{LM}^{b}$ & JM & K L & $\mathrm{JL}$ & KM & $\mathrm{JL}$ & $\mathrm{JM}$ & $\mathrm{JL}$ & $\mathbf{K L}$ & $\mathbf{K L}$ & KM & \\
\hline
\end{tabular}

$a$ : Sérum produit entre deux porcs Pitman-Moore.

$b$ : Génotype déduit.

dentes. Les sérums informatifs se groupent de manières différentes que pour la famille I, ce qui s'explique par leur polyvalence. On retrouve quatre classes de porcelets et le génotype de la truie non typée est déduit aisément de ceux des porcelets et du verrat. 
L'interprétation génétique, selon laquelle un seul système régit tous les facteurs mis en évidence, a été régulièrement vérifiée dans toutes les familles typées. Les allosérums ne possèdent apparemment pas d'anticorps contre un système différent non lié avec le système étudié.

\section{Rôle du système lymphocytaire sur la durée de vie des greffes cutanées}

L'étude du rôle possible de ce système sur la survie d'allogreffes cutanées a été réalisée dans la fratrie 2 . Les Io porcelets de race Large White étaient âgés de 4 mois et n'avaient subi aucune intervention antérieure.

Dans cette expérience, nous avons utilisé une technique de greffe différente de celle mise en œuvre pour immuniser. En effet, la durée de survie des greffes de grande taille, $I_{5}$ à $20 \mathrm{~cm}^{2}$, est difficile à apprécier de manière précise et la réalisation d'un nombre élevé de telles greffes nécessiterait beaucoup de temps. I,a technique employée est celle décrite pour les rongeurs (BILIINGHAM et SiI,VERS, I96I) qui avait déjà été expérimentée avec succès chez le Porc (BAKER et ANDRESEN, I964). I1 s'agit de greffes totales dermo-épidermiques, circulaires de $15 \mathrm{~mm}$ de diamètre non suturées maintenues en place dans le lit du greffon par un pansement compressif.

Le prélèvement du greffon se fait à l'aide d'un emporte-pièce. Par simple pression et 3 ou 4 mouvements de rotation alternatifs, on découpe la peau sur 3 à $4 \mathrm{~mm}$ d'épaisseur. Il suffit ensuite de déliter le greffon du conjonctif graisseux sous-jacent à l'aide d'un bistouri. Les greffes ont toutes été faites sur les faces latérales du thorax et le pansement protecteur était maintenu par un bandage adhésif faisant le tour du corps. Cette technique de greffe permet de réaliser près de 20 à 25 greffes en une heure par deux personnes. La cicatrisation est excellente mais le risque d'arrachage avant cicatrisation, en dépit du pansement est de io p. Ioo environ.

Le porc Large White dont 1'épiderme est clair permet des observations macroscopiques précises. Pour évaluer la durée de survie des greffes, nous avons pris les critères établis pour l'estimation de l'évolution des allogreffes cutanées chez 1'Homme. (CONVERSE et RAPAPORT, I956). La date du rejet appréciée macroscopiquement, correspond au point de vue fonctionnel à l'arrêt de la circulation sanguine dans le greffon. Celui-ci est considéré comme rejeté lorsque après être passé par un stade de coloration rouge vif, avec le plus souvent un bourrelet réactionnel périphérique, il devient cyanotique, brillant, oedémateux et collant au doigt. Ce stade évolutif est un critère précis car la transformation se fait en 24 heures, de manière constante.

Chaque porc a reçu une greffe, en triple exemplaire d'un ou plusieurs de ses frères et trois autogreffes témoins. Il a été fait au total II 4 greffes dont 84 allogreffes qui représentent 28 combinaisons différentes. Une combinaison correspond aux trois greffes d'un donneur sur un receveur. Le tableau 3 résume le schéma d'immunisation et la durée de vie des greffes.

L'état des greffes a été suivi quotidiennement à partir du $5^{\mathrm{e}}$ jour. Treize greffes ont été exclues de l'analyse ; douze étaient arrachées dans les premiers jours (4 autogreffes et 8 allogreffes), la treizième présentait un point de suppuration. Les ror greffes restantes ont très bien cicatrisé.

Pour chaque combinaison, la survie calculée est la moyenne des 3 greffes, mais généralement, les greffes d'un même donneur ont subi le rejet le même jour. Dans un cas, la survie est déduite à partir d'une seule greffe. L'état des autogreffes était 
TABLEAU 3

Relations génétiques entre les donneurs et les receveurs pour chacune des 28 combinaisons de greffes dans la famille $n^{\circ} 2$ et les survies respectives de ces greffes

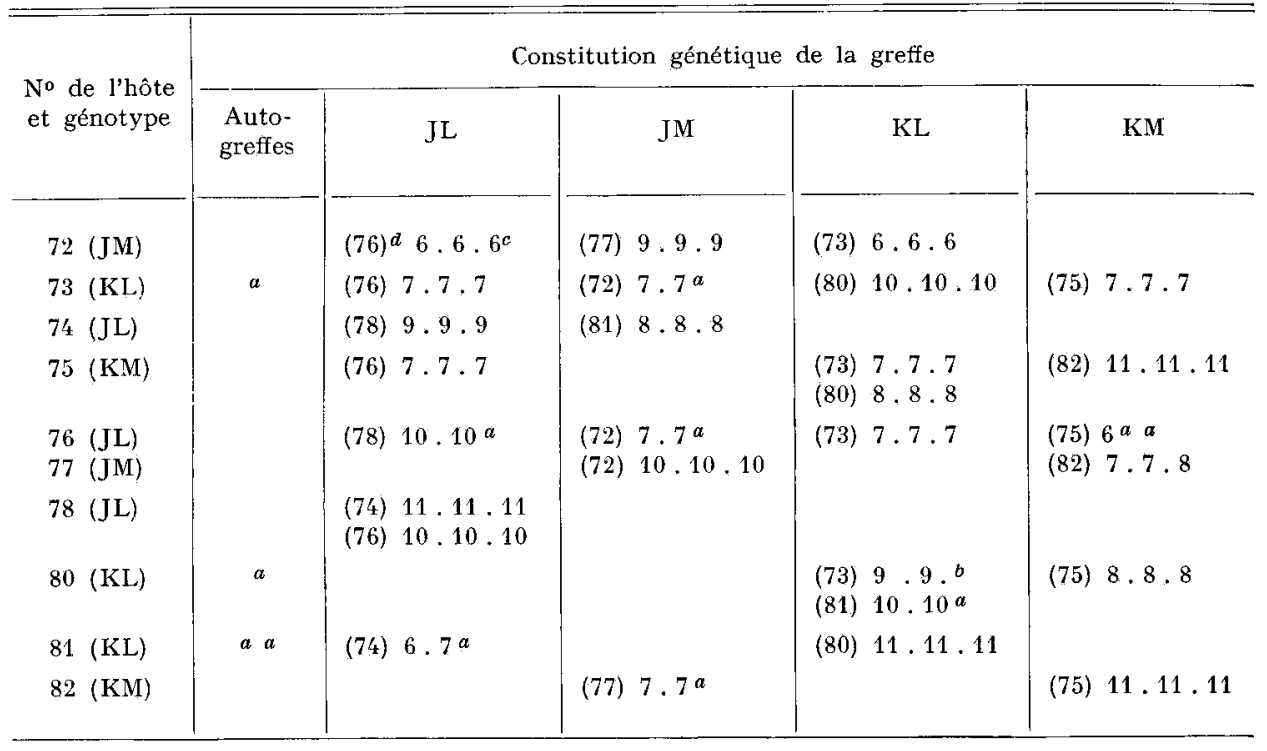

a) Greffes arrachées.

b) Greffes infectées.

c) Survie en jours.

a) Numéro d'identification du donneur.

\section{TABLEAU 4}

Durée de vie moyenne des greffes de peau dont les combinaisons sont groupées en fonction des génotypes respectifs du donneur et du receveur

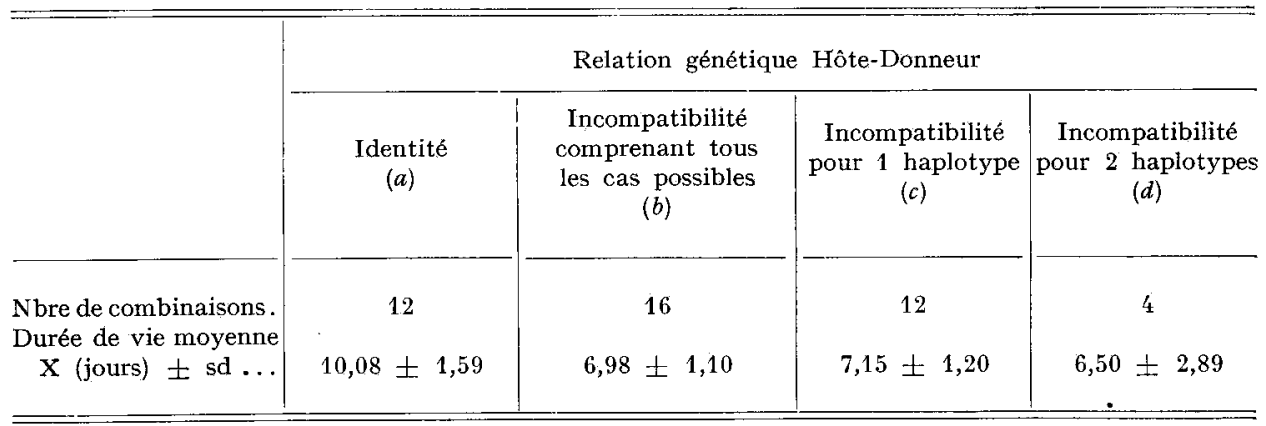

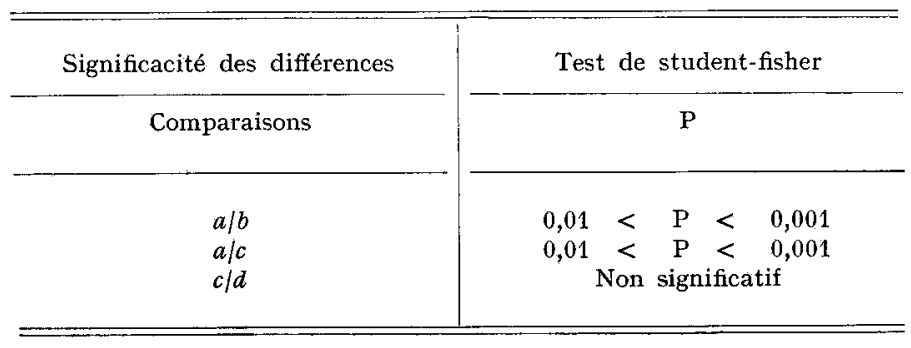


parfait au bout d'un mois. Les résultats de cette étude sont résumés dans le tableau 4 .

L'étude statistique montre que les différences observées dans la survie entre les combinaisons de porcs identiques, c'est-à-dire où le donneur et le receveur ont le même génotype pour le système en cause, et les combinaisons non identiques sont très significatives. L'écart entre les combinaisons qui diffèrent pour un chromosome et celles qui diffèrent par les deux ne l'est pas, mais il est possible que des résultats significatifs puissent être obtenus avec des effectifs plus importants.

Cette expérience démontre que la sélection basée sur le système lymphocytaire assure une survie prolongée des allogreffes cutanées. En tout état de cause, le système lymphocytaire représente un système d'histocompatibilité chez le Porc. On peut être surpris du faible allongement de survie, en valeur absolue, que procure l'identité entre frères ; mais les expériences similaires que nous avons répétées sur d'autres lots de porcs frères ont confirmé ces résultats.

Le rejet précoce des greffes incompatibles ne paraît pas influencer le devenir des greffes compatibles. Ainsi la durée de vie des greffes des porcs 74 et 76 faites chez le porc 78 , tous les trois identiques, n'est pas allongée par rapport à des greffes compatibles chez un hôte ayant reçu en même temps des greffes de donneurs non identiques.

Nous avons désigné ce premier système d'histocompatibilité du porc SL-A par analogie avec la désignation du système HL-A de 1'Homme et en tenant compte de la dénomination zoologique de l'espèce Sus scrofa.

\section{DISCUSSION}

L'immunisation des porcs par des allogreffes cutanées a permis dans la majorité des cas d'induire la production d'anticorps lymphocytotoxiques. Le moment d'apparition des anticorps est variable d'un animal à un autre et ne dépend pas nécessairement du degré de parenté entre le donneur et le receveur. Ainsi, certains porcs immunisés avec un frère ont répondu beaucoup plus rapidement que d'autres qui avaient reçu des greffes de porcs non apparentés. La réponse est dissymétrique chez deux couples de frères, un partenaire répondant normalement alors que l'activité sérique de 1'autre n'a jamais dépassé 25 ou 30 p. Ioo de lyse pour les lymphocytes du donneur, malgré plusieurs rappels. Il pourrait s'agir de différences de force antigénique selon le sens de l'immunisation, cependant, d'autres couples représentant les mêmes constitutions génétiques en présence, se sont stimulés de manière normale. Cela a pu être observé dans les cas où plusieurs groupes de frères typés d'une même fratrie ont été immunisés. Il pourrait s'agir de variations sous la dépendance d'autres facteurs, indépendants du système étudié.

Dans une paire de frères, aucun des deux n'a élaboré d'anticorps, même à un titre faible, au moins pour la technique de cytotoxicité et ceci, malgré 6 greffes de peau successives réparties sur 5 mois. Le typage rétrospectif de ces deux animaux a montré qu'ils étaient identiques pour le système lymphocytaire étudié. Nous avons depuis constamment vérifié que des frères identiques pour ce système, lorsqu'ils sont greffés entre eux, ne produisent jamais d'anticorps décelables par la technique 
de lymphocytotoxicité. Ce résultat est intéressant car il concorde avec le fait que chez 1'Homme seul, les anticorps anti HL-A apparaissent après immunisation.

L'activité sérique se conserve bien dans la majorité des cas. Nous avons cependant observé en quatre occasions, une perte totale d'activité pour des allosérums congelés à $-30^{\circ} \mathrm{C}$, après un délai de I mois. Ce manque de stabilité lors de la conservation a été également noté pour certains allosérums anti-tissus de la Souris (AMos, I962) de même que pour les anticorps dirigés contre les antigènes d'histocompatibilité faibles dans cette espèce (HIIDEMANN, I970). Aucune explication n'a été fournie pour ce phénomène.

Au début de notre expérience, nous immunisions les porcs de la même fratrie, au hasard. A présent, les paires sont constituées de manière à ne différer que pour un seul chromosome, ce qui limite le nombre de spécificités différentes entre le donneur et le receveur. Malgré cette précaution, les allosérums produits ne sont pas, en général, très spécifiques. Une étude en cours sur le degré de polyvalence d'un certain nombre de nos réactifs apportera des précisions sur ce sujet. La difficulté que nous rencontrons à produire des réactifs purs même lorsque les deux frères d'une paire ne diffèrent que par un chromosome, permet de penser que le système est complexe. Cette complexité ne doit pas surprendre si l'on admet que le système d'histocompa-

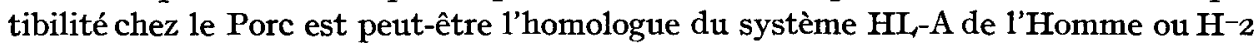
de la Souris. Or, le polymorphisme du système $\mathrm{H}^{-2}$ de la Souris est remarquable (GRAFF, I970) et il en est de même pour le système HL-A (Amos, I969). Dans chacun des deux systèmes, les antigènes d'histocompatibilité sont sous la dépendance d'une région chromosomique comprenant au moins 6 " subloci " pour la souris (SHREFFLER et KLEIN, I970), et au moins 2 (DAUSSET et al., I968) peut-être 3 " subloci " pour l'Homme (WALFORD et al., I969). On peut supposer qu'il en est de même pour le Porc.

La différence de survie des allogreffes de peau entre porcs identiques et non identiques pour le système lymphocytaire SL-A est significative bien que relativement limitée, en valeur absolue. Ce résultat contraste avec ce que l'on observe pour 1'homme, où fréquemment deux frères $\mathrm{HL}_{1}-\mathrm{A}$ identiques acceptent les greffes de peau réciproques pour une durée de 20 à 25 jours (Amos et al., I968). (CEPPEI_LINI et al., I969). Chez le Chien, quelques expériences dans ce domaine font apparaître également des survies nettement allongées (CoHEN et Kozaxi, I969). Par contre, dans certaines combinaisons, chez le Rat, les greffes de peau entre animaux H-I compatibles ne survivent que 4 jours de plus que chez des rats $\mathrm{H}$-I incompatibles (BILDSOE, I970). I1 est possible que les allogreffes cutanées chez le Porc et dans d'autres espèces, résistent moins bien aux réactions de rejet de l'hôte. En faveur de cette hypothèse, on peut remarquer le rejet rapide des allogreffes primaires de peau entre porcs non apparentés en 5 à 8 jours (BINNs, I967), (Lucke et al., I968), alors que chez 1'Homme, la survie moyenne des greffes de peau primaire est de io à I2 jours (CEPPELLINI et al., I969) pour des individus pris au hasard. Enfin, les porcs d'une même fratrie, SL-A incompatibles se comportent comme des porcs non apparentés, ce qui est également le cas pour l'Homme (CEPPELLINI et al., I969).

Tous les résultats obtenus chez le Porc ne concordent cependant pas avec les nôtres. Ainsi, des survies de I4 à 20 jours ont été observées (SAISON et INGRAM, I963) entre porcs différents pour plusieurs facteurs érythrocytaires étudiés ; mais un point important est le critère de rejet retenu. 
La survie relativement courte des greffes de peau entre frères SL-A identiques, pourrait aussi être due à la présence d'autres systèmes forts d'histocompatibilité non détectés. Il semble néanmoins que le système SL-A présente toutes les caractéristiques d'un système majeur si on prend comme référence le système HL-A de 1'Homme. Seuls, les antigènes du système SL-A se sont montrés jusqu'ici capables de susciter la production d'anticorps sériques, tout au moins avec nos moyens d'investigations. Des résultats d'expériences poursuivies au laboratoire (non publiés), à 1'aide de la technique de cultures lymphocytaires mixtes, confirment bien que seul, le système SL-A est capable de provoquer une transformation blastique, ce qui est en accord avec son rôle dominant dans le rejet des greffes.

L'existence d'anticorps sériques contre les antigènes SL-A seuls et la stimulation lymphocytaire par ce système confirment 1'hypothèse selon laquelle SL,A est en tous points l'homologue des systèmes HL-A de 1'Homme (Amos et BACH, I968a) $\mathrm{H}^{-2}$ de la Souris (Hodes et SVEDMYR, I970) ou encore H-I du Rat (WIISON, I967).

Il est possible que dans un autre système expérimental que la greffe de peau, par exemple, la greffe rénale ou la greffe d'autres organes, l'influence du système SL-A sur la survie puisse être beaucoup plus nette. Enfin on peut noter que le système SL-A ségrège indépendamment des facteurs érythrocytaires étudiés, qui ne représentent cependant qu'une fraction des systèmes érythrocytaires connus chez le Porc. La connaissance du système SL-A devrait permettre la clarification du problème actuellement posé par la tolérance parfois remarquable du Porc aux allogreffes hépatiques (TERBLANCHE et al., I968). (CALNE et al., I969) faites jusqu'ici sans test d'histocompatibilité. Dans un premier stade, seul le typage des fratries est envisageable, mais à condition de ne pas prendre d'élevages consanguins, les différents degrés de compatibilité entre porcelets frères représentent peut-être un matériel expérimental intéressant permettant une extrapolation à l'Homme.

\section{CONCLUSION}

Le système lymphocytaire allotypique SL-A, mis en évidence chez le Porc représente le premier système d'histocompatibilité identifié dans cette espèce. Il se comporte par son influence sur la durée de vie des allogreffes de peau, sa complexité, son immunogénicité et aussi par sa capacité de stimuler les lymphocytes in vitro, comme le système majeur et doit être considéré comme l'homologue des systèmes majeurs décrits dans les autres espèces. Ceci confirme que dans chaque espèce animale, il y a certainement un système majeur dont le rôle physiologique n'est pas encore entièrement compris (DAUSSE'T et RAPAPOR'T, Ig68), bien que son rôle dans le rejet des allogreffes soit essentiel.

Rę̧u pour publication en octobre 1970.

\section{REMERCIEMENTS}

Nous remercions le docteur Luba Podiachouk qui a assuré la détermination des groupes sanguins de nos animaux et M. GROSCLAUDE qui a bien voulu discuter notre manuscrit. 


\title{
SUMMARY
}

\section{DETERMINATION OF A HISTOCOMPATIBILITY SYSTEM}

\author{
IN THE PIG. (THE SL-A SYSTEM)
}

The current investigation was undertaken in order to determine one or more histocompatibility systems in the pig. Lymphocytotoxic antibodies were obtained from $\mathrm{I}_{7}$ pairs of sibs and 6 pairs of unrelated Large White pigs, and 2 pairs of half sib Pitman Moore pigs given reciprocal immunosuppression by skin grafting. 70 Large white pigs from 5 families were tested with 461 mphocytotoxic antibodies by means of Terasaki's microdroplet technique. Within each family, parent characteristics as detected by antibodies were transmitted to the progeny according to simple Mendelian proportion, as if depending on one locus. 4 groups of piglets were obtained every time. The factors determined by this system are co-dominant and irrespective of sex. Genetic identity for the lymphocyte system between two littermates allows a significant 3 -day survival of skin allografts. This lymphocyte system therefore appears to be a histocompatibility system with characteristics similar to the HL-A system for man. It is likely to represent the major histocompatibility system for the species. We termed it SL-A according to its zoological name : Sus scrofa.

\section{RÉFÉRENCES BIBLIOGRAPHIQUES}

Ameteau J., I97o. Étude de la production d'anticorps antileucocytaires chez le Porc. Observations anatomocliniques et histologiques des allogreffes cutanées. Thèse de doctorat vétérinaire. Paris.

Amos B. D., r962. Isoantigens of mouse red cells. Ann. N. Y. Acad. Sci., 97, 69-82.

amos B., Ward F. E., Zmijewski C. M., Hattler B. G., Seigler H. F., ig68 a. Graft donor selection based upon single locus (Haplotype) analysis within families. Transplantation, 6, 524-534.

Amos D. B., BAch F. H., I968 b. Phenotypic Fxpressions of the Major Histocompatibility locus in Man (HL-A): leukocyte antigens and mixed leucocyte culture reactivity. J. Exp. Med., 128, 623-637.

Amos D. B., 1969. Genetic and antigenic aspects of human histocompatibility systems. Adv. Immunol., 10, $25 \mathrm{I}$.

Baker L. N., ANDREsen E., I964. Skin grafting in pigs : evidence for a histocompatibility mechanism. Transplantation, 2, II8-1 I9.

Bildö̈̈e P., Sörensen S. F., Pettirossi O., Simonsen M., I97o. Heart and Kidney Transplantation from segregating hybrid to parental cells. Tranpl. Rev., 3, 36-45.

Billingham R. E., Silvers W. K., I96I. Transplantation of tissues and cells, I vol., I49 pages. The Wistar Institute Press. Philadelphie.

Binns R. M., 1967. Bone marrow and lymphoid cell injection of the pig Fetus resulting in transplantation tolerance or immunity, and immunoglobulin production. Natuve, 214, I79-I8I.

Calne R. Y., Selles R. A., Pena J. R., Davis D. R., Millard R. R., Herbertson B. M., Binns R. M., Davies D. A L., I969. Induction of Immunological tolerance by porcine liver allofgrafts. Nature, 223, $472-476$.

Ceppellini R., Mattiuz P. L., Scudeller G., Visetti M., I969. Experimental allotransplantation in man: I. The role of the HL-A system in different genetic combinations. Transpl. proc., 1, 385-389.

CoHeN I., KOzAKI M., I969. The production of Isoantibodies in littermates dogs after allogenic skin grafting. Transplantation, $y, 468-474$.

Converse J. M., Rapaport F. T., 1956. The vascularization of skin autografts and homografts. An Experimental study in man. Ann. Surgery, 143, 306-315.

Dausser J., Rapaport F. T., I968 a. Human Transplantation, 383-393, Grune et Stratton. N. Y. London.

Dausset J., Colombani J., Legrand L., Feingold N., ig68 b. Le deuxième sub-locus du système HL-A. Nouvelle Revue Franf. Hêmatol., 8, 841-846.

Ferrones S., Tosi R. M., Centis D., 1967. Anticomplementary factors Affecting the lymphocytotoxicity Test, 357-364. Histocompatibility Testing. Munksgaard. Copenhagen.

Graff R. J., 1970. Polymorphism of histocompatibility genes in the mouse. Transpl. Proc., II, I5-23.

Herberman R. B., Ig69. Cytolysis of human cells by antibodies in normal rabbit serum. Transplantation, 8, 813-820. 
Hildemann W. H., I970. Strong and weak histocompatibility antigens. General discussion. Transpl. Rev., 3, 92 .

Hodes R. J., Svedmyr E. A. J., I97o. Specific cytotoxicity of H-2 incompatible mouse lymphocytes following mixed culture in vitro. Transplantation, $\mathbf{9}, 470-477$.

Kourilsky F., I966. Étude sur la composition allo-antigénique du leucoblaste de la leucémie aiguë par la méthode de cytotoxicité in vitro. Thèse Doctorat Médecine, Paris.

Lovelock J. E., Bishop M. W. H., I959. Prevention of freezing damage to living cells by DMSO. Nature, 183, I394-1395.

Lucke J. N., Immelman E. J., Srmes M. O., Hunt A. C., I968. Use of horse anti-pig leucocyte serum to suppress the homograft reaction in pigs. Nature, 217, 560 .

Medawar P. B., I958. The homograft reaction. Proc. Roy. Soc. London, 149, B, I45-r66.

Mittal K. K., Mickey M. R., Singal D. P., Terasaki P. I., I 968 . Serotyping for homotransplantation. XVIT. Refinement of microdroplet lymphocyte cytotoxicity test. Transplantation, 6, 9I3-927.

SAISON R., InGRAM D. G., I963. Production of specific hemagglutinins in pigs after receiving skin homografts. Nature, 197, 296-297.

ShrefFler D. C., KLein J., i97o. Genetic organization and gene action of mouse H-2 region. Transpl. Proc., II, 5 .

Stetson C. A., Demopoulos R., I958. Reactions of skin homografts with specific immune sera. Ann. N. Y. Acad. Sci., '73, 687-692.

Terblanche J., Peacock J. M., Hobbs K. E. F., Hunt A. C., Bowes J., Tierris E. J., Palmer D. B., Blecher 'T. E., 1968. Orthotopic liver homotransplantation : an experimental study in the unmodified pig. S. Afric. Med. J., 42, 486-497.

Vatman M., Renard Christine, Nizza P., r970. Technique de préparation de suspensions pures de lymphocytes à partir de sang de porc. Rev. Europ. Études Clin. Biol., 15, 572-575.

WAlford R. M., Gallagher R., SJAARDA J. R., 1964. Serologic typing of human lymphocyte with immune sera obtained after homografting. Science, 144, 868-870.

Walford R. L., Finkelstein S., Hanna C., Collins Z., I969. Third sublocus in the HL-A human transplantation system. Nature, 224, 74-75.

Wilson D. B., 1967. Quantitative studies on the mixed lymphocyte interaction in rats. I. Conditions and parameters of response. $J$. Exp. Med., 126, 625-654. 